\title{
Peters anomaly in PHACE syndrome
}

Nadine M. Shabeeb, MPH, ${ }^{a}$ David A. Plager, MD, ${ }^{b}$ and Anita N. Haggstrom, MD $^{c}$

${ }^{a}$ Indiana University School of Medicine, Indianapolis, Indiana; ${ }^{b}$ Department of Ophthalmology, Indiana University Health, Indianapolis, Indiana; ${ }^{c}$ Department of Dermatology, Indiana University Health, Indianapolis, Indiana

Submitted September 16, 2016.

Revision submitted January 15, 2017.

Correspondence: Anita Haggstrom, MD, 550 N. University Blvd, UH 3240, Indianapolis, IN 46220 (email: ahaggstr@iu.edu).

\section{Word count: 1,138}

This is the author's manuscript of the article published in final edited form as:

Shabeeb, N. M., Plager, D. A., \& Haggstrom, A. N. (2017). Peters anomaly in PHACE syndrome. Journal of American Association for Pediatric Ophthalmology and Strabismus. https://doi.org/10.1016/j.jaapos.2017.04.010 
PHACE syndrome is a rare neurocutaneous disorder, with a complex pathogenesis. It presents with a large facial hemangioma associated with anomalies of the posterior fossa of the brain, arterial anomalies, cardiac anomalies, coarctation of the aorta, and eye anomalies. Ocular abnormalities are rare. We report the first published case of an infant with PHACE syndrome and Peters anomaly.

\section{Case Report}

A 6-week-old girl presented at Indiana University Health, Indianapolis, with a history of left facial erythema that appeared shortly after birth and was initially attributed to birth trauma. During the first few weeks of life, she developed more prominent erythema, scattered erythematous papules, and telangiectasia on the left upper face. The patient was also noted to have a cloudy left cornea and was initially referred to pediatric ophthalmology to rule out glaucoma associated with possible Sturge Weber syndrome. On examination, a large facial hemangioma involving the left upper and lower eyelids causing nearly complete ptosis was present (Figure 1A). Fixation and following acuity was present in the right eye but absent on the left. Complete examination of the right eye, including anterior segment and dilated fundus examination, was normal. The left eye had a dense central corneal opacity approximately $6 \mathrm{~mm}$ in diameter that precluded any view of the pupil, lens, or posterior segment (Figure 1A, inset). Iridocorneal adhesions were noted, with iris strands attached to the peripheral edges of the corneal opacity at the 11:00, 3:00, and 5:00 o'clock positions. B-scan ultrasonography showed a normal posterior segment in the left eye. Ultrasound biomicroscopy was performed to evaluate the anterior segment: no anterior polar or other lens opacity was noted (Figure 1B). Intraocular pressure (IOP) was $8 \mathrm{~mm} \mathrm{Hg}$ in right eye and $11 \mathrm{~mm} \mathrm{Hg}$ in left eye. A diagnosis of Peters anomaly was made. 
Because of the large facial segmental hemangioma, the patient was admitted to the hospital for further workup and treatment. To rule out PHACE syndrome, an echocardiogram and magnetic resonance imaging (MRI) and magnetic resonance angiography (MRA) of the head and neck were performed. Echocardiogram showed a small patent foramen ovale versus secundum atrial septal defect, with left to right shunting. The MRI/MRA showed persistent bilateral tortuosity of the internal carotid arteries, no evidence of intracranial vascular malformations, and multiple hemangiomas of the left face, left orbit, left neck, and left posterior mediastinum. Direct laryngoscopy also showed a subglottic hemangioma.

On admission to the hospital, the patient was treated with prednisolone $2 \mathrm{mg} / \mathrm{kg} /$ day and ranitidine $7.5 \mathrm{mg} / \mathrm{kg} /$ day until cerebral imaging could rule out critical stenosis and dysplasia of the intracranial vasculature before starting propranolol. Symptoms of worsening gastroesophageal reflux necessitated increasing ranitidine to $10 \mathrm{mg} / \mathrm{kg} / \mathrm{day}$. The patient experienced respiratory stridor at the outset of admission, and MRI/MRA of the head and neck could not be performed under sedation until hospital day 6 . At that time oral propranolol was initiated at a dosage of $0.5 \mathrm{mg} / \mathrm{kg} / \mathrm{day}$ and titrated to $2 \mathrm{mg} / \mathrm{kg} /$ day. Prednisolone was continued at $2 \mathrm{mg} / \mathrm{kg} / \mathrm{day}$, and the IOP was not monitored daily.

The ptosis completely resolved, and the hemangioma became flat, with residual telangectasias. The patient was weaned off prednisolone over the subsequent 4 months. At 1 year of age the propranolol was also weaned, but cessation resulted in the development of inspiratory stridor attributed to rebound growth of the subglottic hemangioma. Propranolol was restarted, and at 1 year of age the dosage of propranolol remained $1.45 \mathrm{mg} / \mathrm{kg} /$ day.

The patient underwent a surgical procedure to sever the iridocorneal adhesions at the age of 2 months, and she continues to be followed by both dermatology and ophthalmology. The 
corneal opacity has cleared to the point where a faint red reflex is present, and no glaucoma has developed. In view of the right eye being normal, a conservative approach has been taken with regards to surgery of the involved eye; specifically, no penetrating keratoplasty has been recommended.

\section{Discussion}

PHACE syndrome (OMIM 606519) is a rare neurocutaneous disorder, with a complex pathogenesis. It presents with a large facial hemangioma $(\geq 5 \mathrm{~cm})$ associated with anomalies of the posterior fossa of the brain, arterial anomalies, cardiac anomalies, coarctation of the aorta, and eye anomalies. A multicenter study assessing 108 patients with large facial hemangiomas $\geq 22 \mathrm{~cm}^{2}$ reported that nearly $1 / 3$ had PHACE syndrome, and most demonstrated at least one extracutaneous finding. ${ }^{1}$ The most common manifestation was arterial anomalies of the cerebrovasculature (91\%), followed by cardiac anomalies (67\%). Ocular abnormalities were relatively uncommon, seen in only 8 patients $(7.4 \%) .{ }^{1}$ Among the diagnostic criteria for PHACE syndrome, ocular anomalies include persistent fetal vasculature, persistent hyperplastic primary vitreous, retinal vascular anomalies, morning glory disk anomaly, optic nerve hypoplasia, and peripapillary staphyloma. ${ }^{1}$ Other ocular anomalies are considered minor criteria and include: sclerocornea, cataract, coloboma, and microphthalmia. ${ }^{1}$ Most eye findings seen in PHACE are secondary to the primary vascular and neurologic anomalies. ${ }^{2}$ These patients have an increased risk for amblyopia resulting from occlusive, strabismus, or refractive etiologies. ${ }^{3}$ To our knowledge, this is the first reported case of a child with PHACE syndrome and Peters anomaly.

Peters anomaly is a congenital central corneal opacity, with corresponding defects in the posterior stroma, endothelium and Descemet's membrane. ${ }^{4}$ Iridolenticulocorneal adhesions of variable degree are also present. ${ }^{5}$ The corneal endothelial layer, which is absent in Peters 
anomaly, is derived from neural crest cells. The absence of this layer in Peters anomaly is believed to be from defective neural crest cell migration, because they fail to reach the central cornea. ${ }^{5}$ Given that anomalies of the anterior circulation outnumber posterior circulation anomalies, it is hypothesized the etiology of PHACE syndrome is due to neural crest cells. ${ }^{6}$

Peters anomaly can be seen alone, with other ocular malformations, or with anomalies of well-defined syndromes. ${ }^{7}$ Midline body structures are often involved; common anomalies are cleft lip/palate, congenital heart defects, genitourinary abnormalities, pilonidal cysts, spina bifida, sacral hypoplasia, anal atresia, and vesicocolonic fistula. ${ }^{7}$ Peters-plus syndrome is defined as Peters' anomaly, congenital cardiac defects, craniofacial dysplasia, cleft lip/palate, skeletal changes, and developmental delay. ${ }^{4}$

Our patient demonstrated Peters anomaly ipsilateral to segmental facial hemangioma and cerebrovascular anomalies indicating a potential "field effect," given the proximity of the findings. The association of Peters anomaly and PHACE syndrome may be a coincidental finding in our patient; however, the spatial relationship to a segmental hemangioma distributed in the frontotemporal region that corresponds to patterns of neural crest migration suggests a causal relationship. Winter and colleagues ${ }^{6}$ have hypothesized that the insult causing PHACE syndrome occurs during the first trimester of pregnancy, given the developmental timing of the involved embryonic structures. Similarly, the critical event leading to Peters anomaly is believed to occur in the first trimester, when the anterior chamber is formed. ${ }^{8}$ Further study is warranted to understand this potential association.

\section{Literature Search}

PubMed was searched in March and August 2016 for English-language articles using combinations of the following terms: PHACE syndrome, Peters anomaly, anterior segment 
dysgenesis, and infantile hemangioma. 


\section{References}

1. Haggstrom AN, Garzon MC, Baselga E, et al. Risk for PHACE syndrome in infants with large facial hemangiomas. Pediatrics 2010:126; e418.

2. Metry DW, Garzon MC, Drolet BA, et al. PHACE syndrome: current knowledge, future directions. Pediatr Dermatol 2009: 26(4); 381-398.

3. Kronenberg A, Blei F, Ceisler E, Steele M, Furlan L, Kodsi S. Ocular and systemic manifestations of phaces (posterior fossa malformations, hemangiomas, arterial anomalies, cardiac defects and coarctation of the aorta, eye abnormalities, and sternal abnormalities or ventral developmental defects) syndrome. J AAPOS 2005:9;169-73.

4. Harissi-Dagher M, Colby K. Anterior segment dysgenesis: peters anomaly and sclerocornea. Int Ophthalmol Clin 2008:48;35-42.

5. Kivlin JD, Fineman RM, Crandall AS, Olson RJ. Peters' anomaly as a consequence of genetic and nongenetic syndromes. Arch Ophthalmol 1986:104;61-4.

6. Winter PR, Itinteang T, Leadbitter P, Tan ST. PHACE syndrome-clinical features, aetiology and management. Acta Paediatr 2016;105:145-53.

7. Traboulsi EI, Maumenee IH. Peters' anomaly and associated congenital malformations. Arch Ophthalmol 1992: 110; 1739-41.

8. Genetic and Rare Diseases Information Center. Peters anomaly. 2015. https://rarediseases.info.nih.gov/diseases/7377/peters-anomaly. Accessed December 29, 2016. 


\section{Legends}

FIG 1. A, Clinical photograph of 6-week-old girl at presentation showing large, segmental leftsided facial hemangioma causing ptosis; there was a dense central corneal opacity in the left eye (inset). B, Ultrasound biomicroscopy image showing iridocorneal adhesions of Peters anomaly in the left eye. 


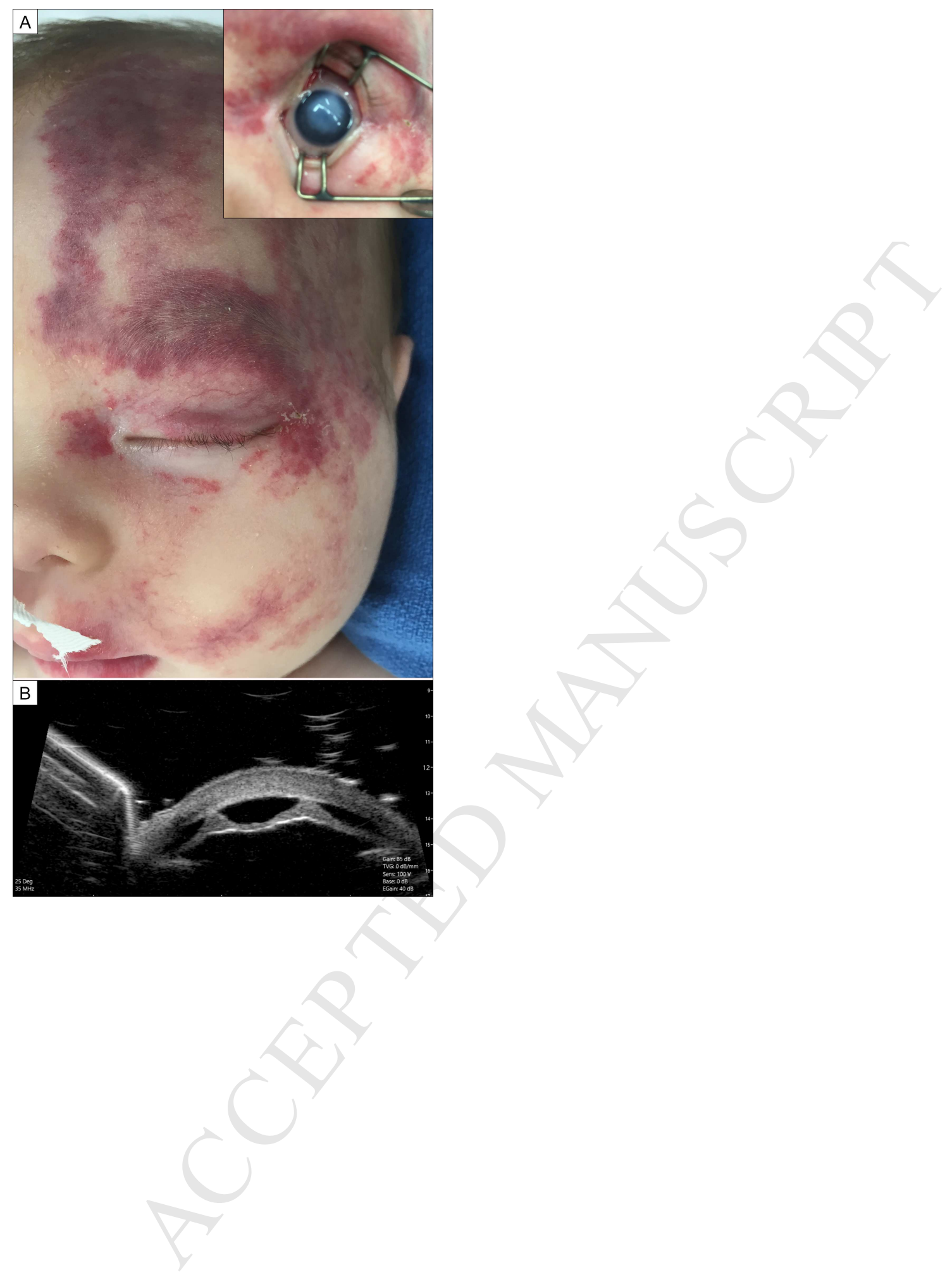

